\title{
Uso de estrogênio e progesterona na terapia hormonal pós-menopausa: revisão sistemática de sua influência no domínio cognitivo
}

\author{
Estrogen and progesterone in postmenopausal hormone therapy: a systematic review of influence \\ in cognitive domain \\ Uso de estrógeno y progesterona en la terapia hormonal posmenopáusica: revisión sistemática de su \\ influencia en el dominio cognitivo
}

Recebido: 31/08/2021 | Revisado: 09/09/2021 | Aceito: 11/09/2021 | Publicado: 13/09/2021

\author{
Isadora Abreu de Carvalho Melo \\ ORCID: https://orcid.org/0000-0001-5879-0058 \\ Universidade Federal de Campina Grande, Brasil \\ E-mail: isaabreumelo@gmail.com \\ Priscylla Emylly Lacerda de Sousa \\ ORCID: https://orcid.org/0000-0001-7338-738X \\ Universidade Federal de Campina Grande, Brasil \\ E-mail: priscyllaemy@gmail.com \\ Ana Janaina Jeanine Martins de Lemos Jordão \\ ORCID: https://orcid.org/0000-0002-3640-1450 \\ Universidade Federal de Campina Grande, Brasil \\ E-mail: janainajeanine@yahoo.com.br
}

\begin{abstract}
Resumo
O objetivo deste artigo foi identificar possível padrão de resposta cognitiva associada a suplementação de estrogênio e/ou progesterona em mulheres após a menopausa. Foi realizada uma revisão sistemática da literatura utilizando o protocolo PRISMA, de corte transversal e análise quanti-qualitativa nas bases de dados PubMed e Scientific Electronic Library Online (SCIELO) utilizando os seguintes Descritores em Ciências da Saúde (DeCS) "Cognição" e "Terapia de reposição hormonal" em inglês e seus sinônimos. Foram identificados 349 artigos que após a aplicação dos filtros resultaram 82 publicações. Não houve duplicidade entre as bases pesquisadas. Após a exclusão de estudos com animais, revisões de literatura e aqueles que a Terapia de Reposição Hormonal foi utilizada com outras finalidades que não o tratamento dos sintomas da menopausa restaram 09 artigos a serem analisados. A terapia hormonal influenciou negativamente a orientação, raciocínio abstrato, volume cerebral e perguntas, além de exacerbar efeitos negativos do Diabetes Mellitus sobre a cognição. Porém, nos domínios atenção, velocidade de processamento, memória operacional e verbal houve uma influência positiva mostrando que as mulheres que utilizaram terapia hormonal obtiveram desempenho melhor que as que nunca utilizaram. Diante dos resultados conflitantes, faz-se necessários mais estudos que avaliem a influência das diferentes formulações, além disso, os fatores de risco para declínio cognitivo devem ser levados em consideração no momento da indicação de terapia hormonal, pois ainda que o tratamento não seja indicado para fins cognitivos, o declínio deste pode causar grande impacto na qualidade de vida das mulheres.
\end{abstract}

Palavras-chave: Saúde da mulher; Cognição; Menopausa; Terapia de reposição hormonal.

\begin{abstract}
The objective this article is identify the cognitive response possible pattern associated the estrogen and/or progesterone supplementation in postmenopausal women. Was carried a systematic literature review using the PRISMA protocol and quantitative-qualitative analysis in the PubMed and Scientific Electronic Library Online (SCIELO) databases using the Health Sciences Descriptors (DeCS) "Cognition" and "Hormone replacement therapy" in English and synonyms. Were identified 349 articles and after apply the filters were resulted 82 publications. There was no duplication between the surveyed databases. After excluding animal studies, literature reviews and articles that Hormone Replacement Therapy was used for purposes other than the treatment of menopausal symptoms, there were still 09 articles to be analyzed. The hormone therapy negatively influenced in orientation, abstract reasoning, brain volume and questions, also exacerbating the negative effects of Diabetes Mellitus on cognition. However, in the domains attention, processing speed, working and verbal memory was a positive influence, showing that women who used hormone therapy performed better than those who never used it. In view of the conflicting results, further studies are needed to assess the influence of different formulations, in addition, the risk factors for cognitive decline should be taken into account when indicating hormonal therapy, because even if the treatment does not is indicated for cognitive purposes, its decline can have a great impact on women's quality of life.
\end{abstract}

Keywords: Women's health; Cognition; Menopause; Hormone replacement therapy. 


\begin{abstract}
Resumen
El objetivo de este artículo fue identificar un posible patrón de respuesta cognitiva asociado con la suplementación con estrógenos y o progesterona en mujeres posmenopáusicas. Se realizó una revisión sistemática de la literatura utilizando el protocolo PRISMA, análisis transversal y cuantitativo-cualitativo en las bases de datos PubMed y Scientific Electronic Library Online (SCIELO) utilizando los Descriptores de Ciencias de la Salud (DeCS) "Cognición” y "Terapia de Reemplazo Hormonal” en inglés y sus sinónimos. Se identificaron 349 artículos, luego de aplicar los filtros resultaron 82 publicaciones. No hubo duplicación entre las bases de datos encuestadas. Después de excluir los estudios en animales, las revisiones de la literatura y aquellos en los que la Terapia de Reemplazo Hormonal se utilizó para fines distintos al tratamiento de los síntomas de la menopausia, aún quedaban por analizar 09 artículos. La terapia hormonal influyó negativamente en la orientación, el razonamiento abstracto, el volumen cerebral y las preguntas, además exacerba los efectos negativos de la diabetes mellitus en la cognición. Sin embargo, en los dominios atención, velocidad de procesamiento, memoria de trabajo y verbal, hubo una influencia positiva. Dados los resultados contradictorios, se necesitan más estudios para evaluar la influencia de diferentes formulaciones, además, se deben tener en cuenta los factores de riesgo para el deterioro cognitivo a la hora de indicar la terapia hormonal, ya que incluso si el tratamiento no está indicado con fines cognitivos, su declive puede tener un impacto importante en la calidad de vida de las mujeres. Palabras clave: Salud de la mujer; Cognición; Menopausia; Terapia de reemplazo de hormonas.
\end{abstract}

\title{
1. Introdução
}

A menopausa é definida como o fim do período fértil feminino, sendo considerada em menopausa uma mulher após um ano de amenorreia constatada de forma retrospectiva (Bacon, 2017). Trata-se de um evento fisiológico com alterações endócrinas permanentes marcadas por secreção reduzida dos hormônios ovarianos, estrogênio e progesterona, que ocorre geralmente em torno dos 40 aos 58 anos, sendo considerada precoce quando antes dos 45 (Anagnostis et al., 2019; Cui et al., 2019; Nelson, 2008).

Os sintomas da menopausa afetam aproximadamente 75\% das mulheres (Kim et al., 2019). São relatados sintomas vasomotores, ressecamento vaginal, diminuição da libido, insônia, fadiga, dor nas articulações e nos músculos (Lund et al., 2018; Santoro et al., 2015). Além disso, muitas mulheres se queixam de alterações na função cognitiva, humor, sono e comportamento que têm relação direta com o declínio hormonal que ocorre durante o processo de envelhecimento (Gava et al., 2019; Melo et al., 2017).

O estrogênio interage com os sistemas colinérgico e serotoninérgico, que são os principais envolvidos no funcionamento cognitivo normal (Santoro et al., 2015). A maioria relata piora da memória verbal (aprendizagem e recordação da lista de palavras), atenção, velocidade de processamento, organização, planejamento e concentração (Melo et al., 2017).

A avaliação das funções cognitivas inclui um histórico completo fornecido pelo paciente e, de preferência, familiares próximos, além de uso de medicamentos, histórico médico e história familiar. O exame físico, incluindo um exame neurológico e avaliação cognitiva também deve ser realizado (Santoro et al., 2015). O teste cognitivo mais utilizado como triagem para comprometimento cognitivo é o mini exame do estado mental (MEEM) que testa uma ampla gama de funções, incluindo orientação, recordação, atenção, cálculo, manipulação da linguagem e práxis construtiva (Rech et al., 2019). São esperados melhores resultados se níveis mais elevados de instrução e menores se consumo de bebidas alcoólicas e/ou fumo (Bojar et al., 2016; Melo et al., 2017).

A terapia hormonal $(\mathrm{TH})$ na menopausa tem o estrogênio como principal componente reconhecido para o tratamento dos sintomas, seja sob a forma de estrogênios equinos conjugados (CEEs), estrogênios conjugados sintéticos, $17 \beta$-estradiol micronizado, estriol, valerato de estradiol ou hemi-hidrato de estradiol (Gambacciani et al., 2018). Em mulheres com um útero intacto a progesterona (hidroxiprogesterona, progesterona micronizada ou acetato de medroxiprogesterona (MPA)) também deve fazer parte da TH (Stute et al., 2016; Palacios; Mejía, 2016). As recomendações devem basear-se em diretrizes atualizadas (Gambacciani et al. 2018; Lumsden et al., 2016; Baber et al., 2016).

Estudos sugerem que a TH pode proporcionar efeitos benéficos na cognição, proteção do tecido nervoso e manutenção da homeostase cerebral (Del Río et al., 2020). No entanto, há pesquisas que obtiveram conclusões discordantes como risco 
aumentado para doença de Alzheimer e exacerbação de processos neurodegenerativos (Wu et al., 2020). Variáveis podem alterar os resultados cognitivos obtidos com a TH como o tempo transcorrido entre o início da menopausa e início da terapia, formulações, vias e horários de administração, tempo de uso, além dos fatores relacionados à mulher, nível de escolaridade, condições socioeconômicas, hábitos de vida, presença de condições clínicas não diagnosticadas (Hara et al., 2015).

Diante da inconsistência sobre os benefícios da TH na cognição em mulheres após menopausa, e do crescente envelhecimento populacional associado ao declínio cognitivo fisiológico, a atual pesquisa tem como objetivo identificar possível padrão das respostas quanto aos domínios cognitivos influenciados pela suplementação de estrogênio e/ou progesterona através de revisão integrativa de literatura. Além disso, delinear possível causa do início da terapia hormonal e definir qual o domínio da cognição mais frequentemente citado.

\section{Metodologia}

O estudo foi delineado a partir das etapas descritas a seguir: identificação da questão norteadora; seleção das fontes de pesquisa; seleção dos estudos ao considerar os critérios de inclusão e exclusão; extração dos dados por meio da síntese qualitativa; mapeamento e divulgação dos resultados (Souza et al., 2010). Para a busca foram utilizados os Descritores em Ciências da Saúde (DeCS) "Cognição" e "Terapia de reposição hormonal" em português e inglês e seus sinônimos nas bases de dados PubMed e Scientific Electronic Library Online (SCIELO). No tocante à seleção automática nas plataformas foram aplicados os filtros conforme à Figura 1 .

Figura 1. Filtros aplicados nas bases de dados eletrônicas.

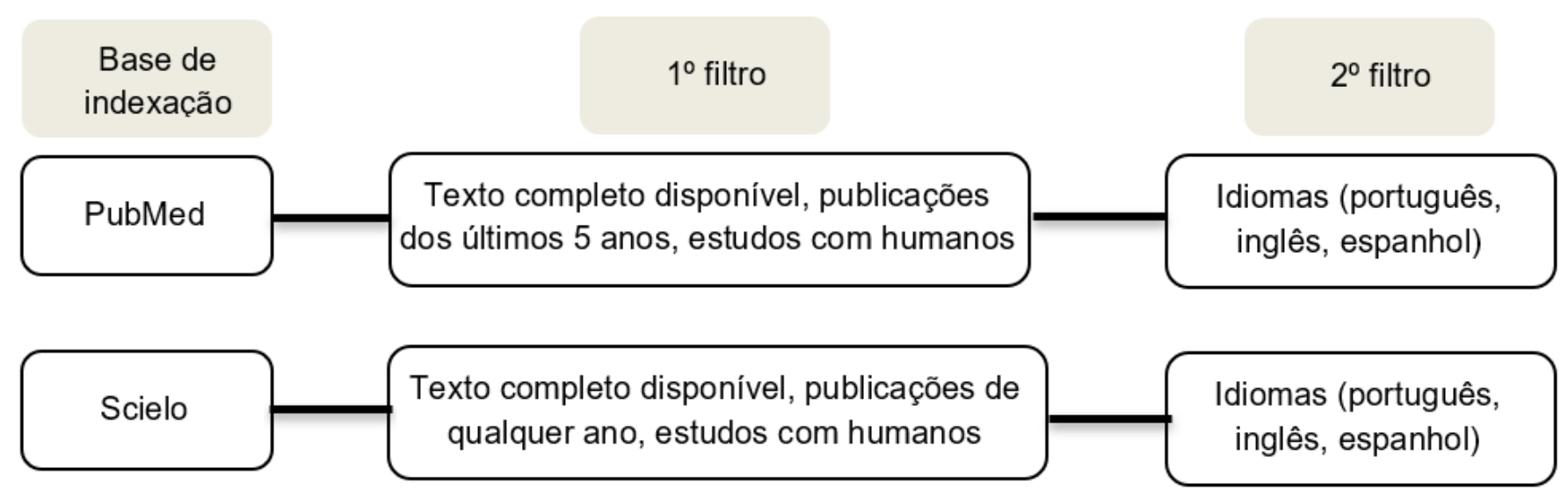

Fonte: Autores.

Para finalizar, a busca foi realizada de forma independente pelos revisores e a análise de concordância foi realizada através do teste Kappa usando o aplicativo BioEstatística V.1.1.0 e calculado de acordo com o método de Landis e Kock (1977), o valor encontrado foi $K=0,88$. As divergências foram solucionadas a partir de um consenso, sem a necessidade de outro revisor.

Este estudo segue a recomendação PRISMA proposta por Moher et al. (2015), contendo fluxograma para apresentação da quantidade de artigos que foram apresentados em cada etapa durante a seleção da amostra, apresentado posteriormente. A elegibilidade dos artigos foi determinada pelo mnemônico Paciente - Intervenção - Comparação - "Outcomes"/desfecho (PICO) (figura 2). Além disso, foram excluídos trabalhos de revisão de literatura, cartas ao editor, opiniões de especialistas e resenhas. 
Figura 2. Critérios de elegibilidade dos artigos.



Os resultados foram apresentados de forma descritiva em duas etapas. A primeira, consistiu na descrição dos artigos selecionados, utilizando-se de tabela, com dados sobre procedência, tipo de estudo, autoria, tamanho das amostras, tipo de TH utilizada e principais conclusões. Posteriormente, avaliou-se variáveis quanto ao tratamento: a formulação da TH, via de administração, tempo de uso; quanto às participantes da pesquisa: nível de escolaridade, idade de início do tratamento, comorbidades associadas e hábitos de vida.

\section{Resultados e Discussão}

A pesquisa identificou 349 artigos, desses 345 na ferramenta de buscas PUBMED e 4 no SCIELO, não houve duplicidade de estudos entre as bases. Após a aplicação dos filtros supracitados restaram 78 publicações na PUBMED que somadas às 4 da SCIELO resultaram em 82 artigos destinados a avaliação através da leitura de títulos e resumos. Os passos que seguiram a aplicação dos filtros, a partir do check-list PRISMA estão descritos na figura 3, seguindo o designado por Page e colaboradores (2021). Ao ler todos os resumos, apenas 17 tratavam sobre terapia hormonal em mulheres após menopausa. Os 17 artigos foram analisados na íntegra para identificação de respostas aos objetivos da atual pesquisa, não obstante apenas 9 contemplavam o estabelecido pela proposta. Dessa forma, baseado no conceito estipulado por Melnyk et al. (2010), foram selecionados estudos com nível de evidência classificados como II, IV e V que correspondem, respectivamente, a ensaio clínico randomizado, caso controle e estudo transversal que foram considerados de relevância e confiáveis para o estudo (Tabela 1). 
Figura 3. Fluxograma da seleção dos estudos construído a partir das designações PRISMA seguindo o seguinte padrão: "Identificação", "Seleção", "Elegibilidade” e "Inclusão” baseado de seleção dos artigos.

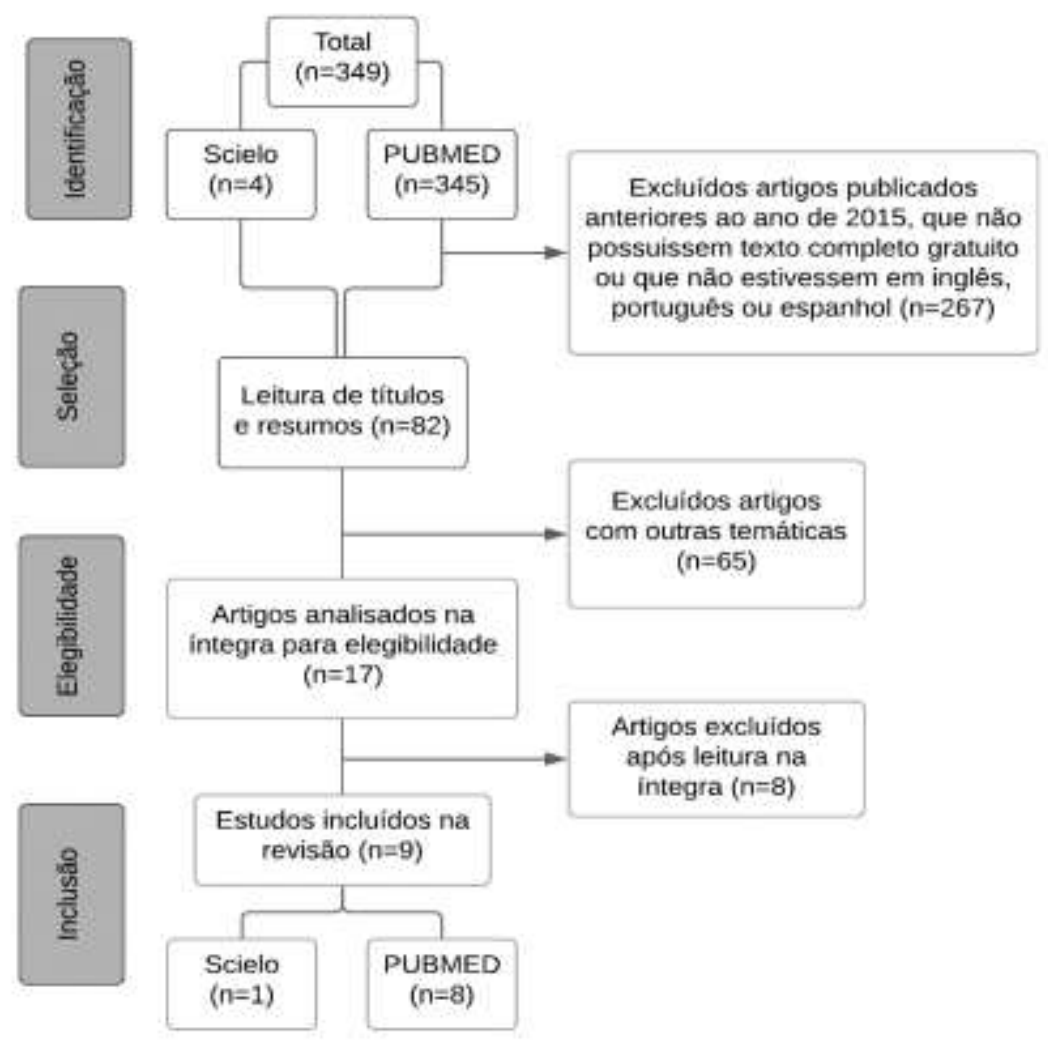

Fonte: Autores.

Tabela 1 - Características dos estudos segundo a identificação, autores, país, ano de publicação, classificação segundo o fator de impacto e nível de evidência.

\begin{tabular}{lcccc}
\hline Autores & País & Ano & Fator de Impacto & Nível de Evidência \\
\hline HOMEZ et al. & Venezuela & 2003 & 4,540 & IV \\
\hline ESPELAND et al. & Estados Unidos & 2015 & 9,901 & II \\
\hline WROOLIE et al. & Estados Unidos & 2015 & 4,105 & II \\
\hline HENDERSON et al. & Estados Unidos & 2016 & 9,901 & II \\
\hline ZHANG et al. & Estados Unidos & 2016 & 2,740 & II \\
\hline GIRARD et al. & Reino Unido & 2017 & 4,130 & II \\
\hline BERENT-SPILLSON et al. & Estados Unidos & 2018 & 3,305 & V \\
\hline MORADI, SADATMAHALLEH, & Irã & 2019 & 1,420 & II \\
ZIAEI & & & & II \\
\hline OBER,SHENAUT, TAYLOR & Reino Unido & 2019 & 1,177 &
\end{tabular}

Fonte: Autores.

Dos nove artigos incluídos, 99\% (n=8) foram em língua inglesa e 11\% (n=1) em espanhola. A idade das participantes nos estudos variou de 41 a 84 anos, sendo a média da variação entre as idades de 17,6 anos. Quanto ao tempo transcorrido entre o diagnóstico da menopausa e início da TH, 11,1\% (n=1) estabeleceu como limite máximo dez anos, 11,1\% (n=1) definiu que a 
TH deveria ser iniciada em até dois anos da menopausa, 22,2\% (n=2) dividiram os grupos em menos de dez e mais de dez anos para início da terapia hormonal e 55,6\% $(n=5)$ foram indiferentes.

No que se refere a presença de comorbidades das mulheres avaliadas, 33,3\% (n=3) excluíram participantes com doenças clinicamente diagnosticadas, $11,1 \%(\mathrm{n}=1)$ não citaram aspectos clínicos da amostra, 55,6\% $(\mathrm{n}=5)$ aceitaram mulheres com alguma comorbidade clínica, dentre essas a mais prevalente foi a hipertensão arterial sistêmica (HAS) presente em $80 \%$ (n=4). No que concerne a presença do alelo da apolipoproteína $\mathrm{E} 4,33,3 \%(\mathrm{n}=3)$ dos estudos incluíram portadoras desse alelo.

No que tange aos principais resultados encontrados (Tabela 2) foi percebido um padrão na análise que aponta que $77,8 \%$ $(n=7)$ dos estudos não encontraram benefícios da terapia hormonal no tocante à cognição. Desses, em 42,8\% (n=3) o uso da terapia foi indiferente em relação ao controle com placebo, enquanto 57,2\% (n=4) apresentaram prejuízo cognitivo na comparação. Entretanto, os estudos apresentam limitações, como formas heterogêneas de coleta de dados, falta de padronização nos questionários, nos testes aplicados e no tamanho da amostra.

Em relação à via de administração da TH, 88,9\% $(\mathrm{n}=8)$ utilizaram somente via oral enquanto $11,1 \%(\mathrm{n}=1)$ associou via oral e gel vaginal. Quanto ao tratamento, 11,1\% (n=1) utilizaram TH com estrogênio isolado, enquanto 33,3\% (n=3) o utilizaram combinado à progesterona, em 55,6\% $(\mathrm{n}=5)$ foram adotadas as duas possibilidades a depender das necessidades da amostra. Em $66,7 \%(n=6)$, o estrogênio utilizado foi somente Estrogênio Equino Conjugado (EEC) e em 22,2\% (n=2) somente 17 $\beta$-E, em $11,1 \%(n=1)$ os dois puderam ser utilizados. A progesterona utilizada em $100 \%(n=9)$ foi AMP.

Mais de um domínio da cognição foi avaliado em cada trabalho, dessa forma, cinco avaliaram memória verbal, dois memória visual, em um avaliou habilidade de construção, dois avaliaram habilidade de linguagem, três avaliaram atenção, dois avaliaram orientação, dois avaliaram raciocínio abstrato, um avaliou memória operacional, dois avaliaram função executiva, um avaliou visuoespacial e dois avaliaram cognição global. Além disso, três estudos avaliaram possíveis alterações estruturais através de exames de neuroimagem, um trabalho avaliou volume e dois avaliaram a atividade cerebral.

Tabela 2. Distribuição dos artigos selecionados de acordo com a base, tipo de estudo, ano de publicação, autores, amostra, tratamento, tempo e resultados alcançados.

\begin{tabular}{|c|c|c|c|}
\hline $\begin{array}{l}\text { Procedência/ } \\
\text { Tipo de estudo }\end{array}$ & Autoria & Amostra (n)/ Tratamento/Tempo & Resultados \\
\hline $\begin{array}{l}\text { Scielo/ } \\
\text { Investigação } \\
\text { longitudinal } \\
\text { prospectiva caso- } \\
\text { controle }\end{array}$ & $\begin{array}{l}\text { HOMEZ et al., } \\
2003\end{array}$ & $\begin{array}{l}\text { Grupo teste } \mathbf{1}(\mathrm{n}=14) \text { : EEC } 0,625 \\
\text { mg+AMP } 2,5 \mathrm{mg} / \mathrm{dia}, \mathrm{VO} . \\
\text { Grupo teste } \mathbf{2}(\mathrm{n}=22) \text { : EEC } 1.250 \\
\text { mg+AMP } 5,0 \mathrm{mg} / \mathrm{dia}, \mathrm{VO} \text {. } \\
\text { Grupo controle }(\mathrm{n}=36) \text { : placebo, VO. } \\
\text { Tempo: } 12 \text { meses }\end{array}$ & $\begin{array}{l}\text { Não houve diferença nos testes de memória verbal e } \\
\text { visual, habilidade de construção, linguagem e atenção } \\
\text { entre os grupos } 1 \text { e } 2 \text { e seus controles. Contudo, o grupo } \\
\text { teste } 2 \text { piorou nos domínios orientação e raciocínio } \\
\text { abstrato quando comparado ao grupo controle. }\end{array}$ \\
\hline $\begin{array}{l}\text { Pubmed/ Ensaio } \\
\text { Clínico } \\
\text { randomizado } \\
\text { duplo-cego e } \\
\text { controlado por } \\
\text { placebo }\end{array}$ & $\begin{array}{l}\text { ESPELAND et } \\
a l ., 2015\end{array}$ & $\begin{array}{l}\text { Diabéticas: } 538 \text { ( } n=274 \text { teste e } n=264 \\
\text { controle) } \\
\text { Sem diabetes: } n=6696(n=3282 \text { teste e } \\
n=3413 \text { controle) } \\
\text { Intervenção: EEC } 0,625 \mathrm{mg} \text { isolado ou } \\
\text { em combinação com AMP } 2,5 \mathrm{mg} \text {, VO. } \\
\text { Tempo: } 6 \text { anos }\end{array}$ & $\begin{array}{l}\text { Os níveis mais altos de estrogênio decorrentes da TH } \\
\text { exacerbaram os riscos que o diabetes tipo } 2 \text { representa } \\
\text { para a piora da função cognitiva em mulheres } \\
\text { menopausadas }\end{array}$ \\
\hline $\begin{array}{l}\text { Pubmed/ Ensaio } \\
\text { clínico } \\
\text { randomizado } \\
\text { controlado por } \\
\text { placebo }\end{array}$ & $\begin{array}{l}\text { WROOLIE et } \\
a l ., 2015\end{array}$ & $\begin{array}{l}\text { Grupo teste } \mathbf{1}(\mathbf{n}=\mathbf{1 7}): 17 \beta-\mathrm{E}, \mathrm{VO} \text {. } \\
\text { Grupo teste } \mathbf{2}(\mathbf{n}=\mathbf{1 3}) \text { : EEC, VO. } \\
\text { Grupo controle }(\mathbf{n}=\mathbf{2 4}) \text { : interrupção do } \\
\text { uso de TH e substituição por placebo. } \\
\text { Tempo: } 2 \text { anos }\end{array}$ & $\begin{array}{l}\text { Houve melhora no desempenho nos testes de atenção, } \\
\text { memória operacional e velocidade de processamento nos } \\
\text { grupos } 1 \text { e } 2 \text { em comparação ao grupo controle. Para a } \\
\text { memória verbal pontuações mais altas foram obtidas } \\
\text { pelo grupo teste } 1 \text {. }\end{array}$ \\
\hline
\end{tabular}




\begin{tabular}{lll}
\hline Pubmed/ & HENDERSON & Grupo teste $(\mathbf{n = 2 8 4}): 17 \beta-\mathrm{E} 1 \mathrm{mg} / \mathrm{dia}$ \\
Ensaio Clínico & et al., 2016 & VO $\pm 45 \mathrm{mg}$ progesterona micronizada \\
randomizado, & & gel vaginal a $4 \%$ \\
duplo-cego & & Placebo $(\mathbf{n}=\mathbf{2 8 5})$ \\
controlado por & Sendo 271 menopausa precoce $(<6$ anos $)$ \\
placebo & e 372 tardia $(>10$ anos $)$ \\
& Tempo: 57 meses \\
\hline
\end{tabular}

\begin{tabular}{lll}
\hline Pubmed/ & ZHANG et al., & $\begin{array}{l}\text { Grupo teste }(\mathbf{n}=674): \text { EEC 0,625 } \\
\text { mg/dia isolado ou em combinação com }\end{array}$ \\
Ensaios clínico & 2016 & AMP 2,5 mg/dia, VO. \\
randomizado & & Placebo $(\mathbf{n = 6 9 1})$ \\
controlado por & Tempo: 1,3 anos EEC isolado e 3 anos \\
placebo & EEC + AMP. \\
& \\
\end{tabular}

Grupos teste e controle: indiferente para função executiva, cognição global e memória verbal episódica seja menopausa precoce ou tardia

Pubmed/
Ensaio clínico
randomizado,cruz
ado, duplo-cego e
controlado por
placebo

Houve efeitos adversos nos volumes cerebrais principalmente no cingulado anterior e no giro frontal medial adjacente e no córtex orbitofrontal e risco maior de comprometimento cognitivo no grupo teste em relação ao grupo controle

GIRARD et al., Grupo teste (n=6): 17 $-\mathrm{E} 2 \mathrm{mg} / \mathrm{dia}$ por 11 dias + AMP $100 \mathrm{mg} /$ dia a partir do $12^{\circ}$ dia, VO.

Placebo $(\mathbf{n}=6)$

Tempo: 2 ciclos de 28 dias, totalizando 8 semanas.

$\begin{array}{ll}\text { GIRARD } \text { et al., } & \text { Grupo teste }(\mathbf{n = 6}): 17 \beta-E ~ 2 \mathrm{mg} / \mathrm{dia} \text { por } \\ & 11 \text { dias + AMP } 100 \mathrm{mg} / \mathrm{dia} \text { a partir do } \\ & 12^{\circ} \text { dia, VO. } \\ & \text { Placebo }(\mathbf{n}=\mathbf{6}) \\ & \text { Tempo: } 2 \text { ciclos de } 28 \text { dias, totalizando } 8 \\ & \text { semanas. }\end{array}$

BERENTSPILLSON al., 2018
Grupo 01(n=13): usuárias de TH no et momento do estudo experimental;

Grupo $02(\mathbf{n = 2 5})$ : Usuárias de TH no passado

Ambos utilizaram EEC $0,625 \mathrm{mg} \pm$ AMP, VO.

Grupo 03 (n=19): nunca utilizaram TH. Tempo: mínimo 10 anos.
Houve aumento do recrutamento do córtex pré-frontal dorsolateral durante a troca de tarefas demonstrando efeitos benéficos da TH quando iniciada precocemente no início da pós-menopausa, porém ainda não mensuráveis através do comportamento e não influenciou no tempo de reação ou na taxa de erros durante os testes de avaliação da cognição utilizados.
Transversal
MORADI, SADATMAHA Clínico randomizado
Grupo Caso (n=68): EEC 0,625 mg+AMP 2,5mg/dia + 01 Comprimido de $500 \mathrm{mg}$ de cálcio e 200 UI de vitamina D.

Grupo Controle $(\mathbf{n}=68): \quad 01$ Comprimido de $500 \mathrm{mg}$ de cálcio e 200 UI de vitamina $\mathrm{D}$.

Tempo: 04 meses.
Não houve diferença nas tarefas de recordação e discriminação verbal entre o uso TH no momento do estudo ou no passado.

Houve maior ativação do córtex frontal inferior esquerdo e córtex pré-frontal direito e pior desempenho na memória verbal no grupo $\mathrm{TH}$ em comparação com mulheres que nunca utilizaram.

LLEH, ZIAEI
2019

\section{OBER,} SHENAUT,

Clínico randomizado TAYLOR, longitudinal

\author{
Grupo TH foi dividido em 2 grupos: \\ Grupo Teste $1 \quad(\mathbf{n}=16)$ : EEC 0,625 \\ $\mathrm{mg}, \mathrm{VO}$. \\ Grupo Teste 2 (n=28): EEC 0,625 mg + \\ AMP 2,5 mg/dia, VO. \\ Grupo Placebo $(\mathbf{n = 5 6})$. \\ Tempo: 07 anos.
}

Houve melhora na função cognitiva, visuoespacial/executiva, memória, atenção, linguagem e abstração no grupo TH. Porém, quando comparados os grupos caso e controle, apenas o domínio da memória apresentou diferença significativa. Houve piora da orientação.

\begin{abstract}
Houve melhor desempenho na memória de evocação imediata e tardia de histórias e pior desempenho da recordação imediata da lista de palavras pelo grupo TH em relação ao placebo. A recordação tardia da lista de palavras foi indiferente Houve melhora na recordação tardia da lista de palavras para EEC isolado comparado à associação com AMP.
\end{abstract}

EEC: estrógenos equinos conjugados; AMP: acetato de medroxiprogesterona; 17ß-E: 17ß-estradiol; VO: via oral. Fonte: Autores.

Dessa forma, em 22,2\% (n=2) o uso de TH não exerceu influência sobre os seguintes domínios cognitivos: memória verbal e visual, linguagem, atenção, função executiva, habilidade de construção e cognição global, ambos utilizaram $17 \beta$-E em associação a AMP. Em 11,1\% (n=1) apesar de um resultado global indiferente, o grupo que teve uma dose duplicada de EEC e AMP demonstrou um desempenho pior em raciocínio abstrato e orientação. Exames de neuroimagem foram realizados em 33,3\% $(\mathrm{n}=3)$ dos estudos, foram observados efeitos relacionados à $\mathrm{TH}$ principalmente no córtex frontal como aumento do volume do giro do cingulado anterior e giro frontal medial resultando em piora na função cognitiva, além de uma maior ativação do córtex frontal e pré-frontal sem influência nos resultados da avaliação cognitiva, como pode ser observado abaixo na figura 4 , correspondente a cada autor e seu resultado positivo, neutro ou negativo. 
Em 33,3\% (n=3) observou-se que a TH influenciou negativamente a orientação, raciocínio abstrato, volume cerebral, memória e tempo de formular perguntas, além de exacerbar os efeitos negativos do Diabetes Mellitus (DM2) sobre a cognição das mulheres, em 100\% (n=3) foi utilizado o EEC associado ou não a AMP. Já em 33,3\% (n=3) afirmaram uma interferência positiva nos domínios de atenção, velocidade de processamento, memória operacional e verbal em comparação ao grupo que não utilizou TH, desses em 33,3\% (n=1) pode ser utilizado 17 $\beta$-E ou EEC ambos isolados em menopausa recente e nos demais $(n=2)$ foi utilizado o EEC em associação ou não a AMP, sendo um em menopausa recente e outro em menopausa tardia (Gráfico 1).

Figura 4. Síntese do impacto da terapia hormonal por domínio cognitivo avaliado em Positivo, Negativo e Neutro (Colunas) e Autores citados abaixo da análise em cada linha correspondente.

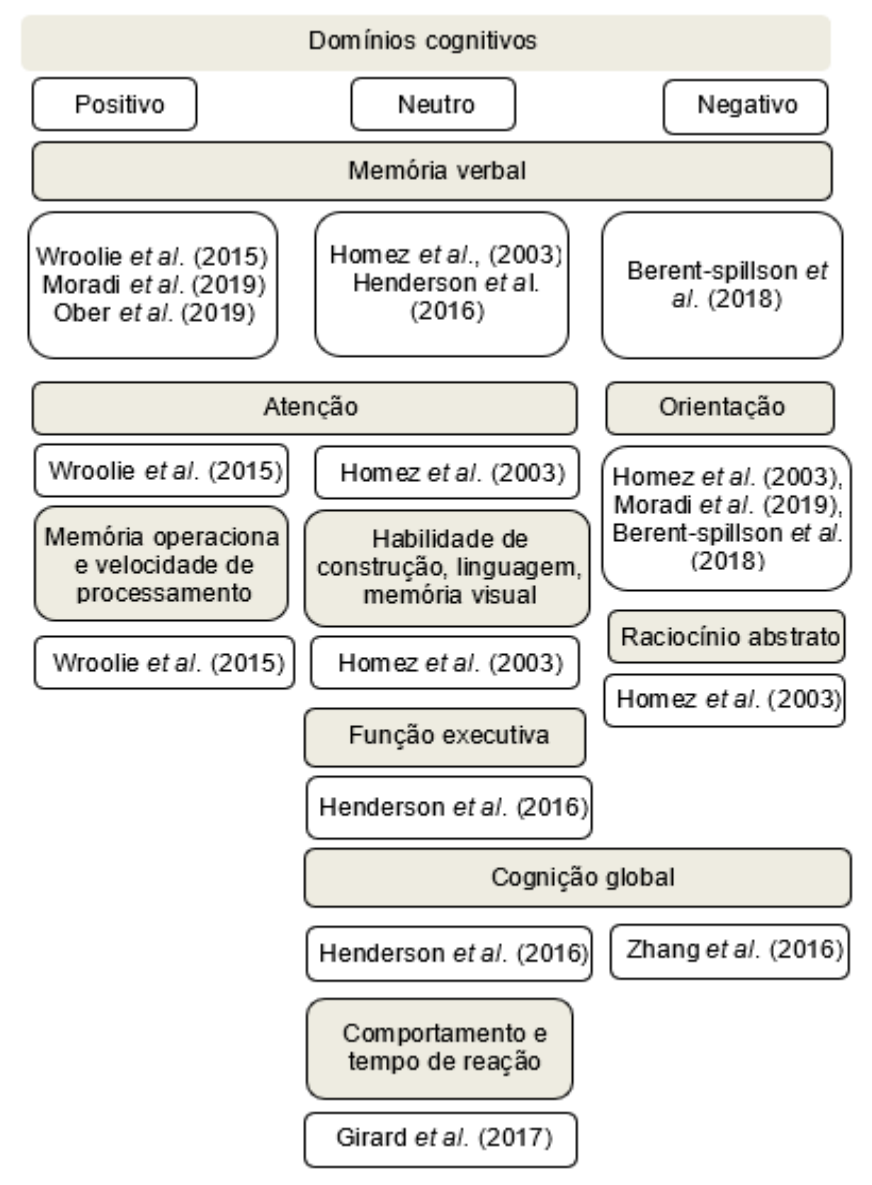

Fonte: Autores. 
Gráfico 1. Síntese do impacto da TH nos domínios cognitivos avaliados de acordo com a terapia utilizada, sendo as barras negativas indicando a quantidade de estudos que avaliaram os resultados da $\mathrm{TH}$ como negativos, as barras positivas indicando a quantidade de estudos que avaliaram os resultados da $\mathrm{TH}$ como positivos e as barras vazadas indicando a quantidade de estudos que avaliaram os resultados da TH como indiferente.

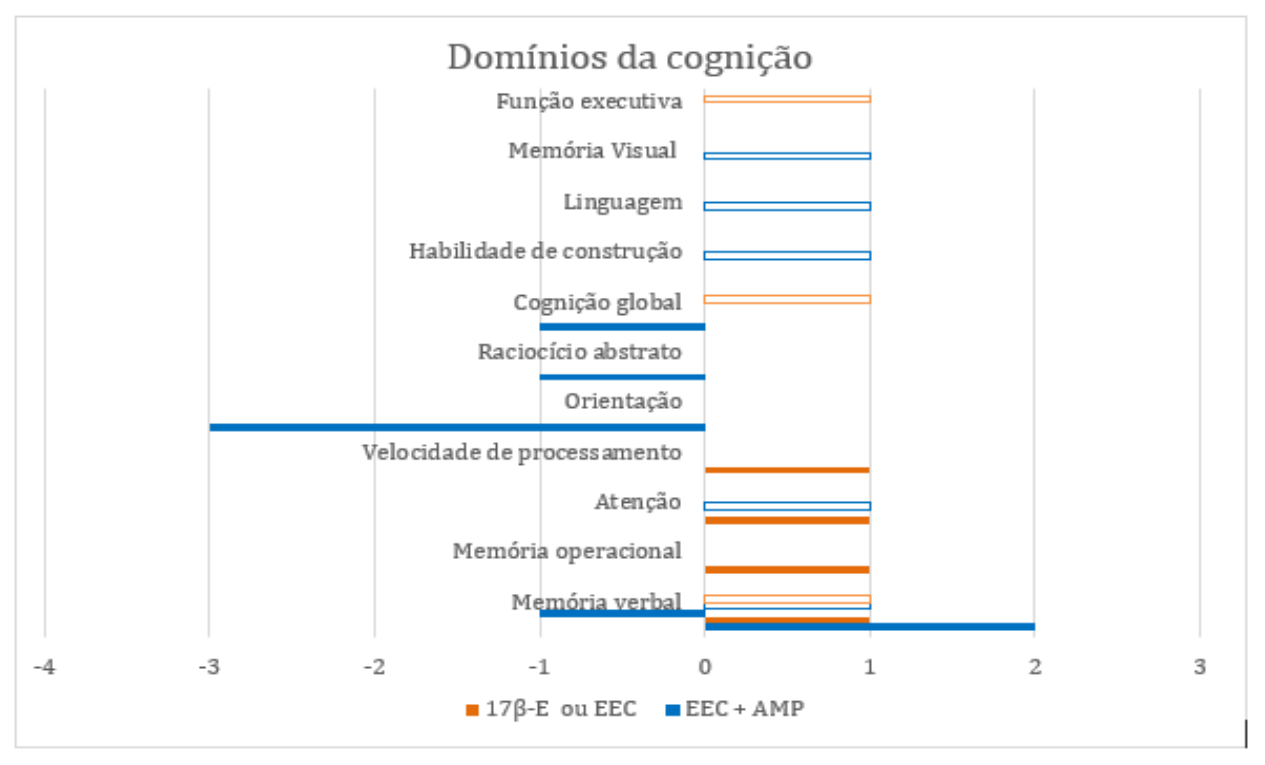

Fonte: Autores.

Tal resultado conflitante também pode ser observado em outras revisões. A terapia com estrogênio é comprovadamente muito eficaz na mitigação dos sintomas da peri-menopausa que, para muitas mulheres, resulta em melhorias significativas na qualidade de vida (Wren, 2009). Porém, alguns estudos relacionaram a terapia hormonal com benefícios cognitivos, enquanto outros a relacionaram com indiferença e até declínio cognitivo (Kantarci et al., 2016). Para Angenendt et al. (2018), o tipo de estrogênio e progesterona poderiam influenciar nos resultados: 17b-estradiol, delta 8,9 dehidroestrona e levonorgestrel podem melhorar o aprendizado e a memória, enquanto estrona, o etinilestradiol, progesterona natural e vários progestágenos sintéticos (acetato de medroxiprogesterona, acetato de noretindrona), porém em nossas análises essa relação não foi observada.

A revisão feita por Petersi et al. (2019), assim como o presente estudo e os anteriormente citados, aponta que as evidências existentes no tocante aos benefícios da TH para a cognição são variadas devido à dificuldade de padronização da amostra, sendo incluídas mulheres com diferentes fatores de risco genéticos para demência. Já para Angenendt et al. (2018) a dificuldade em determinar o papel da TH na saúde do cérebro após a menopausa deve-se ao fato de cada mulher estar submetida a diferentes exposições a hormônios endógenos e exógenos e também à maioria dos estudos não avaliar separadamente o tratamento contendo estrogênio daquele contendo estrogênio associado a progesterona e a contribuição individual de cada hormônio para os resultados cognitivos.

A desconexão entre os efeitos funcionais e estruturais apresentados por exames de imagens e relatados em alguns artigos avaliados por este estudo também esteve presente em outros trabalhos (Kantarci et al., 2016; Resnick et al., 2009; Greenberg et al., 2006). Para Brinton (2016) e Höller (2020), esse fato sugere que o estrogênio esteja potencializando a função das mitocôndrias nas células gliais e a falta de diferença na função cognitiva entre placebo e TH pode ser explicada pela capacidade do cérebro feminino de catabolizar sua substância branca como combustível a ser aproveitado para atender à demanda bioenergética imposta pela cognição normal.

A piora da cognição observada em único estudo incluído nesse trabalho avaliando pacientes diabéticas pode ser decorrente do papel que desempenha o estrogênio ao favorecer o metabolismo da glicose no cérebro (Carcaillon et al., 2014; 
Gupte et al., 2015). Tal observação também foi apontada em outros dois estudos com pacientes diabéticas (Espeland et al., 2015; Witty; Rodgers, 2015; Mccarrey; Resnick, 2015).

Há três formas principais de estrogênio endógeno, sendo o estradiol o principal responsável pelos efeitos observados no sistema nervoso central (Herrera; Mather, 2015). O tempo de exposição ao estrogênio durante a vida da mulher é inversamente proporcional ao risco de desenvolvimento da doença de Alzheimer (DA), ou seja, quanto maior o tempo, menor o risco, porém quando se trata da TH um do fatores que irão influenciar o resultado é o momento de início da reposição, pois os efeitos neuroprotetores estão presentes apenas quando o tratamento é iniciado de forma precoce, quando inserido em fases tardias da menopausa está associada a um risco maior tanto para o desenvolvimento da DA quanto de outras doenças neurodegenerativas (Depypere et al., 2019).

\section{Conclusão}

Não existe um consenso sobre a forma e a intensidade da influência da terapia hormonal sobre a cognição em mulheres pós-menopausa, seus resultados dependem de fatores intrínsecos e extrínsecos que devem ser considerados durante a realização dos estudos. Foi percebido após análise que o domínio cognitivo mais frequentemente citado e influenciado positivamente pela reposição de estrogênio e/ou progesterona é a memória verbal.

A causa mais evidente identificada para o início da terapia hormonal é o alívio de sintomas que acompanham esse período como ondas de calor, ressecamento vaginal e mudanças de humor. Ademais, sugerimos que, se houver indicação para o início de TRH, que seja priorizada em pacientes com Menopausa recente (1-10 anos), objetivando, além dos efeitos conhecidos, melhora na cognição nos domínios atenção, memórias verbal e operacional e velocidade de processamento utilizando EEC com ou sem associação à AMP, mesmo que tal associação traga benefícios cognitivos apenas à memória verbal, em comparação ao EEC isolado, é necessária em mulheres com útero intacto devido aos riscos de hiperplasia endometrial, a qual pode evoluir para o câncer endometrial se utilizado EEC isolado. No entanto, ainda são necessárias mais pesquisas com pareamento e acompanhamento de longo prazo das usuárias, além da padronização das escalas utilizadas para desta forma quantificar quais os domínios cognitivos são mais influenciados pela intervenção.

\section{Referências}

Anagnostis, P., Paschou, S. A., Katsiki, N., Krikidis, D., Lambrinoudaki, I., \& Goulis, D. G. (2019) Menopausal Hormone Therapy and Cardiovascular Risk: where are we now? Current Vascular Pharmacology, 6(17), 564-572. Bentham Science Publishers Ltd. http://dx.doi.org/10.2174 $/ 1570161116666180709095348$.

Baber, R. J., Panay, N., \& Fenton, A. (2016) International Menopause Society: 2016 IMS Recommendations on women's midlife health and menopause hormone therapy. Climacteric. http://dx.doi.org/10.3109/13697137.2015.1129166.

Bacon, J. L. (2017). The Menopausal Transition. Obstetrics and gynecology clinics of North America. 44, 285-296. http://dx.doi.org/10.1016/j.ogc.2017.02.008.

Berent-Spillson, A., Kelley, A. S., Persad, C. C., Love, T., Frey, K. A., Reame, N. E., Koeppe, R., Zubieta, J., \& Smith, Y. R. (2018) Postmenopausal hormone treatment alters neural pathways but does not improve verbal cognitive function. Menopause, 12 (25), 1424-1431. http://dx.doi.org/10.1097/gme.0000000000001157.

Brasil. Ministério da Saúde. (2008). Manual de Atenção à Mulher no Climatério/Menopausa. Editora do Ministério da Saúde. 192 p.

Brinton, R. D. (2009) Estrogen-induced plasticity from cells to circuits: predictions for cognitive function. Trends In Pharmacological Sciences, 4(30), 212-222. http://dx.doi.org/10.1016/j.tips.2008.12.006.

Carcaillon, L., Brailly-Tabard, S., Ancelin, M., Rouaud, O., Dartigues, J., Guiochon-Mantel, A., \& Scarabin, P. (2014). High plasma estradiol interacts with diabetes on risk of dementia in older postmenopausal women. Neurology, 82(6), 504-511. http://dx.doi.org/10.1212/wnl.0000000000000107.

Cui, X., Yu, X., Sun, G., Hu, T., Likhodii, S., Zhang, J. R., Edward, Gao, X., Fan, Z., \& Zhang, W. (2019). Differential metabolomics networks analysis of menopausal status. Plos One, 14(9), 22353. http://dx.doi.org/10.1371/journal.pone.0222353.

Del Río, J. P., Molina, S., Hidalgo-Lanusa, O., Garcia-Segura, L. M., \& Barreto, G. E. (2020). Tibolone as Hormonal Therapy and Neuroprotective Agent. Trends in endocrinology e Metabolism, 20(20). https://doi.org/10.1016/j.tem.2020.04.007. 
Depypere, H., Vierin, A., Weyers, S., \& Sieben, A. (2016) Alzheimer's disease, apolipoprotein E and hormone replacement therapy. Maturitas. 94, 98-105. Elsevier. https://doi.org/10.1016/j.maturitas.2016.09.009.

Espeland, M. A., Brinton, R. D., Manson, J. E., Yaffe, K., Hugenschmidt, C., Vaughan, L., Craft, S., Edwards, B. J., Casanova, R., \& Masaki, K. (2015) Postmenopausal hormone therapy, type 2 diabetes mellitus, and brain volumes. Neurology, 85(13), 1131-1138. http://dx.doi.org/10.1212/wnl.00 00000000001816.

Gambacciani, M., Biglia, N., Cagnacci, A., Caruso, S., Cicinelli, E., Leo, V. de., Carlo, C. di, Farris, M., Gambera, A., \& Guaschino, S. (2018). Menopause and hormone replacement therapy: the 2017 recommendations of the italian menopause society. Minerva Ginecologica, 70(1). http://dx.doi.org/10.23736/S00264784.17.04151-X.

Gava, G., Orsili, I., Alvisi, S., Mancini, I., Seracchioli, R., \& Meriggiola, M. C. (2019). Cognition, Mood and Sleep in Menopausal Transition: The Role of Menopause Hormone Therapy. Medicina (Kaunas). 55(10). https://www.ncbi.nlm.nih.gov/pmc/articles/PMC6843314/.

Girard, R., Météreau, E., Thomas, J., Pugeat, M., Qu, C., \& Dreher, Jean-claude. (2017). Hormone therapy at early post-menopause increases cognitive controlrelated prefrontal activity. Scientific Reports, 7(1), 1. http://dx.doi.org/10.1038/srep44917

Gupte, A. A., Pownall, H. J., \& Hamilton, D. J. (2015). Estrogen: an emerging regulator of insulin action and mitochondrial function. Journal Of Diabetes Research, 1-9. http://dx.doi.org/10.1155/2015/916585.

Hara, Y., Waters, E. M., Mcewen, B. S., Morrison, J. H. (2015) Estrogen Effects on Cognitive and Synaptic Health Over the Lifecourse. Physiol Rev. 95(3), 785-807. Disponível em: https://www.ncbi.nlm.nih.gov/pmc/articles/PMC4491541/. Acesso em: 20 jun 2020.

Henderson, V. W., John, J. A. St., Hodis, H. N., Mccleary, C.A., Stanczyk, F. Z., Shoupe, D., Kono, N., Dustin, L., Allayee, H., \& Mack, W. J. (2016) Cognitive effects of estradiol after menopause. Neurology, 87(7), 699-708, (Wolters Kluwer Health). http://dx.doi.org/10.1212/wnl.0000000000002980.

Herrera, A. Y., \& Mather, M. (2015). Actions and interactions of estradiol and glucorcorticoids in cognition and the brain: Implications for aging women. Neurosci Biobehav Rev. 55, 36-52.

Höller, Y., Butz, K. H. G., Thomschewski, A. C., Schmid, E. V., Hofer, C. D., Uhl, A., Bathke, A. C., Staffen, W., Nardone, R., Schwimmbeck, F. (20 maio 2020). Prediction of Cognitive Decline in Temporal Lobe Epilepsy and Mild Cognitive Impairment by EEG, MRI, and Neuropsychology. Computational Intelligence And Neuroscience, 1-16. http://dx.doi.org/10.1155/2020/8915961.

Homez De Delgado, B., Pino, Gl., \& Maestre De Homez, G. (2003) Efecto de la terapia hormonal de reemplazo sobre la función cognitiva en la menopausia. Rev. Venez. Endocrinol. Metab. 1(3), 12-18. <http://ve.scielo.org/scielo.php?script=sci_arttext\&pid=S1690-31102003000300004\&lng=es\&nrm=iso>.

Kim, H. K., Jeon, S. H., Ryu, K., Kim, T., \& Park, H. (2019). Comparison of the Efficacy of Tibolone and Transdermal Estrogen in Treating Menopausal Symptoms in Postmenopausal Women. Journal Of Menopausal Medicine, 25(3), 123. The Korean Society of Menopause. http://dx.doi.org/10.6118/jmm.19205.

Lund, K. S., Siersma, V. D., Christensen, K. B. Waldorff, F. B., \& Brodersen, J. (2018). Measuring bothersome menopausal symptoms: development and validation of the menoscores questionnaire. Health And Quality Of Life Outcomes, 16(1), 1. Springer Science and Business Media LLC. http://dx.doi.org/10.1186/s12955-018-0927-6.

Lumsden, M. A., Davies, M., \& Sarri, G. (2016) Diagnosis and Management of Menopause. Jama Internal Medicine, 176(8), 1205. American Medical Association (AMA). http://dx.doi.org/10.1001/jamainternmed.2016.2761.

Mccarrey, A. C., \& Resnick, S. M. (2015). Postmenopausal hormone therapy and cognition. Hormones And Behavior, 74, 167-172. Elsevier BV. http://dx.doi.org/10.1016/j.yhbeh.2015.04.018.

Melo, C. S. B. de., Souza, T. S. de., Teodoro, L. I., Legramanti, S., Fanton, S. V., \& Rückl, S. (2017). Declínio cognitivo e perimenopausa: revisão sistemática. Reprodução \& Climatério, 32(2), 132-137. Elsevier BV. http://dx.doi.org/10.1016/j.recli.2016.11.001.

Moradi, F., Jahanian Sadatmahalleh, S., \& Ziaei, S. (2019). The effect of hormone replacement therapy on cognitive function in postmenopausal women: An RCT. International journal of reproductive biomedicine, 16(12), ijrm.v16i12.3682. https://doi.org/10.18502/ijrm.v16i12.3682

Nelson, H. D. Menopause. The Lancet, 371(9614), 760-770. Elsevier BV. http://dx.doi.org/10.1016/s0140-6736(08)60346-3.

Ober, B. A., Shenaut, G. K., \& Taylor, S. L. (2019). Effects of Hormone Therapy on List and Story Recall in Post-Menopausal Women. Experimental Aging Research, 45(3), 199-222. http://dx.doi.org/10.1080/0361073x.2019.1609169.

Palacios, S., \& Mejía, A. (2016). Progestogen safety and tolerance in hormonal replacement therapy. Expert Opinion On Drug Safety, 15(11), 1515-1525. http://dx.doi.org/10.1080/14740338.2016.1223041.

Rech, C. M. Z., Clapauch, R., \& Bouskela, E. (2019). Sexual Function Under Adequate Estrogen Therapy in Women After Oophorectomy Versus Natural Menopause. Journal Of Women'S Health, 28(8), 1124-1132. http://dx.doi.org/10.1089/jwh.2017.6905.

Santoro, N., Epperson, C. N., \& Mathews, S. B. (2015). Menopausal Symptoms and Their Management. Endocrinology And Metabolism Clinics Of North America, 44(3), 497-515. http://dx.doi.org/10.1016/j.ecl.2015.05.001.

Stute, P., Neulen, J., \& Wildt, L. (2016). The impact of micronized progesterone on the endometrium: a systematic review. Climacteric, 19(4), 316-328. http://dx.doi.org/10.1080/13697137.2016.1187123.

Wroolie, T. E., Kenna, H. A., Williams, K. E., \& Rasgon, N. L. (2015). Cognitive Effects of Hormone Therapy Continuation or Discontinuation in a Sample of Women at Risk for Alzheimer Disease. The American Journal Of Geriatric Psychiatry, 23(11), 1117-1126. http://dx.doi.org/10.1016/j.jagp.2015.05.009. 
Research, Society and Development, v. 10, n. 12, e64101220075, 2021

(CC BY 4.0) | ISSN 2525-3409 | DOI: http://dx.doi.org/10.33448/rsd-v10i12.20075

Wu, M., Li, M., Yuan, J., Liang, S., Chen, Z., Ye, M., Ryan, P.M., Clark, C., Tan, S.C., Rahmani, J., Varkaneh, H. K., \& Bhagavathula, A. K. (2020). Postmenopausal hormone therapy and Alzheimer's disease, dementia, and Parkinson's disease: A systematic review and time-response meta-analysis. Pharmacological Research, (155). Elsevier. https://doi.org/10.1016/j.phrs.2020.104693

Zhang, T., Casanova, R., Resnick, S. M., Manson, J. E., Baker, L. D., Padual, C. B., Kuller, L. H., Bryan, R., Espeland, M. A., \& Davatzikos, C. (2016). Effects of Hormone Therapy on Brain Volumes Changes of Postmenopausal Women Revealed by Optimally-Discriminative Voxel-Based Morphometry. Plos One, 11(3), 0150834. http://dx.doi.org/10.1371/journal.pone.0150834 